\title{
Multi-subband effect in spin dephasing in semiconductor quantum wells
}

\author{
M. Q. Weng and M. W. Wu* \\ Hefei National Laboratory for Physical Sciences at Microscale, \\ University of Science and Technology of China, Hefei, Anhui, 230026, China and \\ Department of Physics, University of Science and Technology of China, Hefei, Anhui, 230026, China ${ }^{\dagger}$
}

(Dated: July 6, 2018)

\begin{abstract}
Multi-subband effect on spin precession and spin dephasing in $n$-type GaAs quantum wells is studied with electron-electron and electron-phonon scattering explicitly included. The effects of temperature, well width and applied electric field (in hot-electron regime) on the spin kinetics are thoroughly investigated. It is shown that due to the strong inter-subband scattering, the spin procession and the spin dephasing rate of electrons in different subbands are almost identical despite the large difference in the D'yakonov-Perel' (DP) terms of different subbands. It is also shown that for quantum wells with small well width at temperatures where only the lowest subband is occupied, the spin dephasing time increases with the temperature as well as the applied in-plane electric field until the contribution from the second subband is no longer negligible. For wide quantum wells the spin dephasing time tends to decrease with the temperature and the electric field.
\end{abstract}

PACS numbers: 72.25.Rb, 72.20.Ht, 71.10.-w, 67.57.Lm, 73.61.Ey

Manipulating and transporting electron spins belong to the subjects of intense research in semiconductor spintronics, ${ }^{1,2}$ which aims to incorporate the spin degree of freedom into the traditional electronic devices. To date most of the technical proposals to manipulate the electron spin are either through an applied magnetic field or via a gate voltage that changes the spin-orbital coupling in confined semiconductor structures through the Rashba effect. ${ }^{3,4}$ Recently it has been realized that a strong inplane electric field also provides another path to manipulate the spin dephasing in the presence of the spin-orbital coupling. Rashba and Efros showed that an in-plane ac electric field can manipulate electron spins efficiently. ${ }^{5}$ Pramanik et al. investigated the effect of an in-plane electric field on the spin dephasing in GaAs quantum wire through Monte-Carlo simulation and revealed that the presence of the strong electric field enhances the spin dephasing. ${ }^{6,7}$ A comprehensive many-body investigation of the hot-electron effect on spin precession and spin dephasing due to the strong in-plane electric field in $n$ type GaAs quantum wells (QW's) has been reported recently ${ }^{8}$ in the electric-field and temperature regime where only the lowest subband is occupied. In that investigation all scattering, ie., the electron-electron, electronphonon and electron-impurity scattering is explicitly included and is calculated self-consistently. It is discovered however that the spin dephasing rate decreases with the applied electric field. In the narrow $\mathrm{QW}$, it is also discovered that the spin dephasing rate decreases with temperature. ${ }^{9,10}$

The spin precession and spin dephasing in wide QW'S should show quite different temperature and electric-field dependence as electrons populate more than one subband and therefore experience quite different spin-orbital coupling strength. In the present paper, we study the multisubband effect on the spin precession/dephasing in $n$ type GaAs QW's, where the spin dephasing mainly comes from the D'yakonov-Perel' (DP) mechanism, ${ }^{11}$ with and without the high in-plane electric field. The inclusion of multi subbands allows us to investigate QW's with wide well width and also allows us to study the regime of higher electric field where the single subband model cannot deal with due to the "runaway effect". ${ }^{8,12}$ We reveal the intra- and inter-subband scattering to the spin dephasing times of each subband. The model of our investigation is composed of (100) GaAs QW's of width $a$ with its growth direction along the $z$-axis. An uniform electric field $\mathbf{E}$ and a moderate magnetic field $\mathbf{B}$ are applied along the $x$-axis (Vogit configuration). Due to the confinement of the QW, the momentum states along the $z$-axis are quantized. Therefore the electron states are characterized by a subband index $n$ and a twodimensional wavevector $\mathbf{k}=\left(k_{x}, k_{y}\right)$, together with a spin index $\sigma$. With the DP term (DPT) included, the Hamiltonian of the electron in the QW reads:

$$
\begin{aligned}
H= & \sum_{n \sigma n^{\prime} \sigma^{\prime} \mathbf{k}}\left\{\left(\varepsilon_{n \mathbf{k}}-e \mathbf{E} \cdot \mathbf{R}\right) \delta_{n n^{\prime}} \delta_{\sigma \sigma^{\prime}}+\left[g \mu_{B} \mathbf{B}+\mathbf{h}_{n n^{\prime}}(\mathbf{k})\right]\right. \\
& \left.\cdot \frac{\boldsymbol{\sigma}_{\sigma \sigma^{\prime}}}{2}\right\} c_{n \mathbf{k} \sigma}^{\dagger} c_{n^{\prime} \mathbf{k} \sigma^{\prime}}+H_{I}
\end{aligned}
$$

Here $\varepsilon_{n \mathbf{k}}=\mathbf{k}^{2} / 2 m^{*}+\left\langle k_{z}^{2}\right\rangle_{n} / m^{*}$ is the energy spectrum of the electron with momentum $\mathbf{k}$ and effective mass $m^{*}$ in the $n$-th subband. $\boldsymbol{\sigma}$ are the Pauli matrices. $\mathbf{R}=$ $(x, y)$ represents the position. $\mathbf{h}_{n}(\mathbf{k})$ is the DPT which serves as an effective magnetic field with its magnitude and direction depending on $\mathbf{k}$. It is composed of the Dresselhaus term ${ }^{13}$ and the Rashba term. ${ }^{3,4}$ For GaAs QW, the leading term is the Dresselhaus one which can be written as:

$$
\begin{aligned}
& h_{n n^{\prime}, x}(\mathbf{k})=\gamma k_{x}\left(k_{y}^{2}-\left\langle n\left|k_{z}^{2}\right| n\right\rangle\right) \delta_{n n^{\prime}} \\
& h_{n n^{\prime}, y}(\mathbf{k})=\gamma k_{y}\left(\left\langle n\left|k_{z}^{2}\right| n\right\rangle-k_{x}^{2}\right) \delta_{n n^{\prime}} \\
& h_{n n^{\prime}, z}(\mathbf{k})=\gamma\left\langle n\left|k_{z}\right| n^{\prime}\right\rangle\left(k_{x}^{2}-k_{y}^{2}\right) .
\end{aligned}
$$


In the above, $\left\langle n\left|k_{z}^{2}\right| n\right\rangle$ represents the average of the operator $-\left(\frac{\partial}{\partial z}\right)^{2}$ over the electronic state of the $n$-th subband and is therefore $n^{2}(\pi / a)^{2}$ under the infinite-well-depth assumption and $\left\langle n\left|k_{z}\right| n^{\prime}\right\rangle$ is the matrix element of $-i \partial / \partial_{z}$ between $n$ - and $n^{\prime}$-th subbands. $\gamma$ here is the spinorbital coupling constant. ${ }^{14}$ The interaction Hamiltonian $H_{I}$ is composed of the Coulomb interaction $H_{e e}$, the electron-phonon scattering $H_{p h}$, as well as the electronimpurity scattering $H_{i}$. Their expressions can be found in textbooks. ${ }^{15,16}$

In order to study the hot-electron effect on spin dephasing, we limit our system to a spacial homogeneous one in order to avoid the additional complicity such as charge/spin diffusion. The kinetic Bloch equations in such a system are constructed using the nonequilibrium Green function method with the gradient expansion ${ }^{16}$ and can be written as:

$$
\dot{\rho}_{n \mathbf{k}, \sigma \sigma^{\prime}}-e \mathbf{E} \cdot \nabla_{\mathbf{k}} \rho_{n \mathbf{k}, \sigma \sigma^{\prime}}=\left.\dot{\rho}_{n \mathbf{k}, \sigma \sigma^{\prime}}\right|_{\text {coh }}+\left.\dot{\rho}_{n \mathbf{k}, \sigma \sigma^{\prime}}\right|_{\text {scatt }},
$$

where $\rho_{n \mathbf{k} \sigma \sigma^{\prime}}$ represent the single particle density matrix elements. The diagonal terms describe the electron distribution functions $\rho_{n \mathbf{k}, \sigma \sigma} \equiv f_{n \mathbf{k} \sigma}$. The off-diagonal elements $\rho_{n \mathbf{k}, \frac{1}{2}-\frac{1}{2}}=\rho_{n \mathbf{k},-\frac{1}{2} \frac{1}{2}}^{*} \equiv \rho_{n \mathbf{k}}$ stand for the interspin-band polarizations (spin coherence). ${ }^{17}$ The second terms in the kinetic equations describe the momentum and energy input from the electric field E. $\left.\dot{\rho}_{n \mathbf{k} \sigma \sigma^{\prime}}\right|_{\text {coh }}$ on the right hand side of the equations describe the coherent spin precession around the applied magnetic field $\mathbf{B}$, the effective magnetic field $\mathbf{h}(\mathbf{k})$ from the DPT as well as the effective magnetic field from the electronelectron Coulomb interaction in the Hartree-Fock order. $\left.\dot{\rho}_{n \mathbf{k} \sigma \sigma^{\prime}}\right|_{\text {scatt }}$ denote the electron-impurity, the electronphonons, as well as the electron-electron Coulomb scattering. The electron-electron Coulomb scattering comes from the Coulomb interaction beyond the lowest order, i.e., the Hartree-Fock order. It is noted that in writing the kinetic equations, we have neglected the intersubband coherence $\rho_{n n^{\prime} \mathbf{k} \sigma \sigma^{\prime}}\left(n \neq n^{\prime}\right)$ as these terms are much smaller than the intra-subband spin coherence due to the small "pumping" terms $h_{n n^{\prime}, z}(\mathbf{k})$.

It is seen that all the unknowns to be solved appear in the coherent and the scattering terms nonlinearly. Therefore the kinetic Bloch equations have to be solved selfconsistently to obtain the electron distribution and the spin coherence. By numerically solving the kinetic Bloch equations in the self-consistent fashion, one is able to obtain the temporal evolutions of the electron distribution functions $f_{n \mathbf{k} \sigma}(t)$ and the spin coherence $\rho_{n \mathbf{k}}(t)$. Once these quantities are obtained, all the quantities such as electron mobility $\mu_{n}$, hot-electron temperature $T_{e, n}$ as well as the spin dephasing rate for electrons in each subband can be deduced. For subband $n$, the mobility is given by $\mu_{n}=\sum_{\mathbf{k} \sigma} f_{n \mathbf{k} \sigma} k_{x} /\left[m^{*} E \sum_{\mathbf{k} \sigma} f_{n \mathbf{k} \sigma}\right]$; the electron temperature is obtained by fitting the Boltzmann tail of the electron distribution function; whereas the spin dephasing rate is determined by the slope of the envelope of the incoherently summed spin coherence $\rho_{n}(t)=\sum_{\mathbf{k}}\left|\rho_{n \mathbf{k}}(t)\right| .{ }^{17,18,19}$

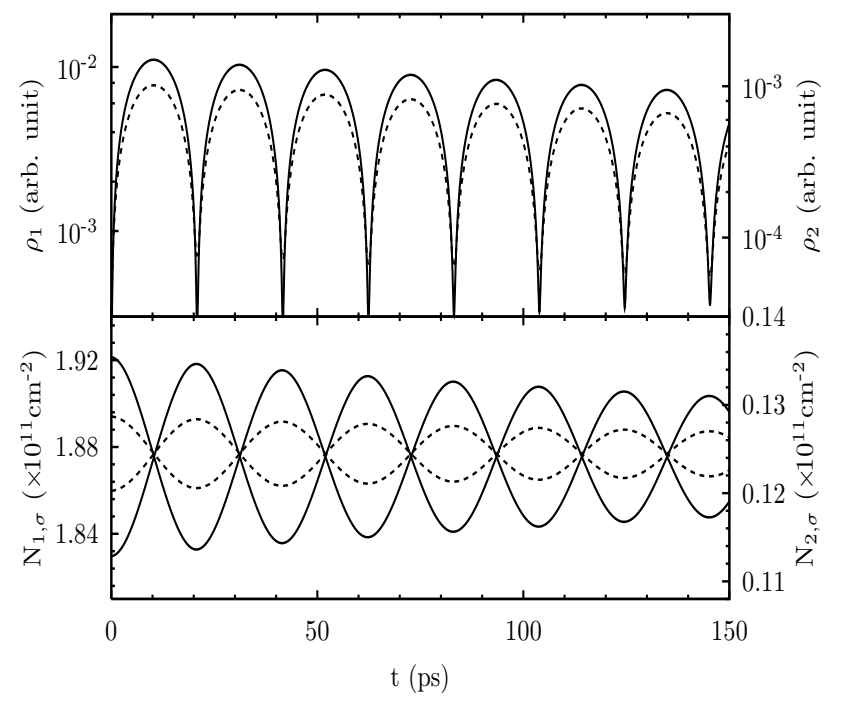

FIG. 1: Temporal evolution of the spin signal with width $a=$ $17.8 \mathrm{~nm}$ and electron density $N_{e}=4 \times 10^{11} \mathrm{~cm}^{-2}$ for $T=200$ $\mathrm{K}$ and $B=4 \mathrm{~T}$ under zero applied electric field. The initial spin polarization $P=2.5 \%$. Upper panel: The incoherentlysummed spin coherence $\rho_{n}$ of $n$-th subband; Lower panel: The electron densities of $n$-th subband with spin $\sigma$. The solid curves are for the first subband and the dashed ones are for the second subband. Note that the scales of the second subband are on the right-hand side of the figure.

The initial conditions at $t=0$ are taken to be $\rho_{n \mathbf{k}}(0)=$ 0 and electron distribution functions are chosen to be those in the steady state under the electric field but without the magnetic field and the $\mathrm{DPT}^{8}$ Specifically $f_{n \mathbf{k}, \sigma}(0)$ is the solution of the kinetic equations (3) in the steady state, with the spin coherence $\rho_{n \mathbf{k}}$, the magnetic field and the DPT set to zero. The implementation schemes of the numerical solution of the kinetic equations and the initial condition can be found in Ref. 8. The total electron density $N_{e}$, the applied magnetic field $B$ and the initial spin polarization $P=\sum_{n \mathbf{k}}\left(f_{n \mathbf{k} \frac{1}{2}}-f_{n \mathbf{k}-\frac{1}{2}}\right) / N_{e}$ are taken to be $4 \times 10^{11} \mathrm{~cm}^{-2}, 4 \mathrm{~T}$ and $2.5 \%$ respectively throughout the paper.

We first study the spin dephasing of electrons in a GaAs QW with $a=17.8 \mathrm{~nm}$. In this QW, the electrons mainly distribute in the first two subbands under the applied electric field up to $3 \mathrm{kV} / \mathrm{cm}$. Therefore, we only consider electrons in the first two subbands with $n$ being 1 and 2 .

We first focus on the temporal evolution of the spin signals. In Fig. 1 we plot the densities of electrons with spinup and -down as well as the corresponding incoherentlysummed spin coherence of each subband versus time $t$ without applied electric field. The background temperature $T=200 \mathrm{~K}$. It is seen from the figure that the electrons in both subbands undergo damped oscillations with a same precession frequency and damping rate. Nevertheless, as $\left\langle 2\left|k_{z}^{2}\right| 2\right\rangle=4\left\langle 1\left|k_{z}^{2}\right| 1\right\rangle$, from Eq. (2) electrons 
with a given wavevector in the second subband experience an effective magnetic field from the DPT with its magnitude three times larger than that of the first subband. At first glance one may expect that the spin dephasing rate of electrons in the second subband should be about 9 times faster than those of electrons in the first subband. It is of particular interest to see that the decay rates of the spin signals of the two subbands are almost identical.

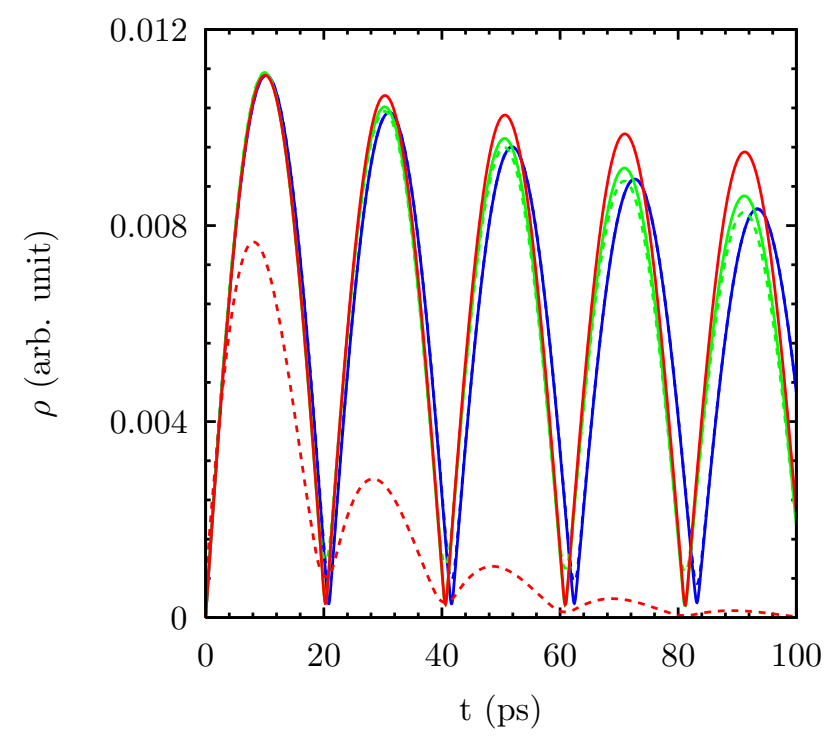

FIG. 2: Temporal evolution of the incoherently-summed spin coherence of each subband $\rho_{n}$ for the same situation in Fig. 1. The blue curves are $\rho_{n}(t)(n=1$ and 2) from the full kinetic equations (Noted that $\rho_{1}(t)$ and $\rho_{2}(t)$ match each other); The green curves are $\rho_{n}(t)$ without the inter-subband Coulomb scattering; The red curves are $\rho_{n}(t)$ without the inter-subband scattering. Solid curves: $\rho_{1}(t)$; Dashed curves: $\rho_{2}(t)$.

In order to reveal the physics of the identicalness of the spin precession and the dephasing of electrons in different subbands, we further plot the temporal evolution of the spin coherence of the above case in Fig. 2. For comparison we also plot $\rho_{n}(t)$ without the inter-subband electronelectron Coulomb scattering (green curves) and those without the inter-subband electron-electron and electronphonon scattering, i.e., without any inter-subband scattering (red curves). It is seen from the figure that if we count for all the scattering, the spin coherence of the different subbands decays identically. However, once the inter-subband of the Coulomb scattering is removed, the spin coherence of the lowest subband decays a little slower and that of the second subband decays a little faster than that with all the scattering included. If one further removes the inter-subband electron-phonon scattering, the spin coherence of the second subband decays very fast and disappears in the first 20 ps while $\rho_{1}$ lasts much longer than the case with all the intersubband scattering included. It is clear to see that as the inter-subband scattering is reduced, the identicalness of the spin dephasing of electrons in different subband is removed. Once the inter-subband scattering is totally removed, the spin coherence differs greatly between the different subbands. Therefore, the identicalness is due to the strong inter-subband scattering.

To further elucidate the effect of the inter-subband scattering on the spin dephasing, we adopt a much simplified model: The system is simply described by the electron number $N_{1}, N_{2}$ and the magnetic momentum $M_{1}$, $M_{2}$ of the two subbands. In the non-degenerate case, the kinetics of the system are: $d N_{i} / d t=\sum_{j=1,2} \alpha_{i j} N_{j}$ and $d\left(N_{i} M_{i}\right) / d t=\sum_{j=1,2} \alpha_{i j}\left(N_{j} M_{j}\right)-N_{i} M_{i} / \tau_{i}^{s}(i=1$, $2)$, with $\alpha_{i j}$ standing for the inter-subband scattering rate and $1 / \tau_{i}^{s}$ for the spin dephasing rate of the $i$-th subband. It is easily seen that in the absence of the inter-subband scattering, the spin signals in the different subbands decay with their own dephasing rate. However, once a strong inter-subband scattering is presented and the electron populations approach to the equilibrium, one is able to obtain that $\alpha_{i i}=-x_{j} / \tau$ and $\alpha_{i j}=x_{i} / \tau$ $(j \neq i)$ from the equilibrium condition $d N_{i} / d t=0$ and the detailed balance condition $\alpha_{i j} N_{j}=\alpha_{j i} N_{i}$ with $x_{i}=N_{i} /\left(N_{1}+N_{2}\right) .1 / \tau$ is the inter-subband relaxation rate. Substituting these relations into the kinetic equations for the magnetic moments $M_{i}$, one gets:

$$
\begin{aligned}
& d M_{1} / d t=-x_{2}\left(M_{1}-M_{2}\right) / \tau-M_{1} / \tau_{1}^{s}, \\
& d M_{2} / d t=x_{1}\left(M_{1}-M_{2}\right) / \tau-M_{2} / \tau_{2}^{s} .
\end{aligned}
$$

In the strong inter-subband scattering limit, i.e., $1 / \tau \gg$ $1 / \tau_{i}^{s}$, the difference of magnetic momentum $M_{1}-M_{2}$ decays with the rate $1 / \tau$. That is the magnetic momentums $M_{i}$ of different subbands become almost identical to each other in the time scale of $\tau$. Taking this fact into account, one easily gets the equation that controls the magnetic momentum $M=M_{1}=M_{2}: d M / d t=-\sum_{i}\left(x_{i} / \tau_{i}^{s}\right) M$. Thus in the strong inter-subband scattering limit, the magnetic momentums of different subbands decay with the same rate $1 / \tau_{s}=\sum_{i} x_{i} / \tau_{i}^{s}$. Therefore, it is understood that, in the presence of the strong inter-subband scattering, electrons hop frequently among the subbands and fast exchange the spin signal. Consequently electrons experience an average DP effective magnetic field of the different subbands during the spin precession and acquire the same spin dephasing rate. Moreover, we also discovered that in the case of strong electric field where the DPT can also change the spin precession rates, ${ }^{8}$ a strong inter-subband scattering also causes the identicalness of the spin precession rates of different subband, regardless from what expected from the DPT analysis that the change of the spin precession rate of the second subband should be 3 times larger than that of the first one.

We then turn to the temperature dependence of the spin dephasing of electrons in QW's with different well width. The spin dephasing time (SDT) versus the background temperature without applied electric field is presented in Fig. 3 for QW's with $a=17.8 \mathrm{~nm}$ and $a=12.6$ $\mathrm{nm}$ respectively. In the figure we also plot the SDT in 


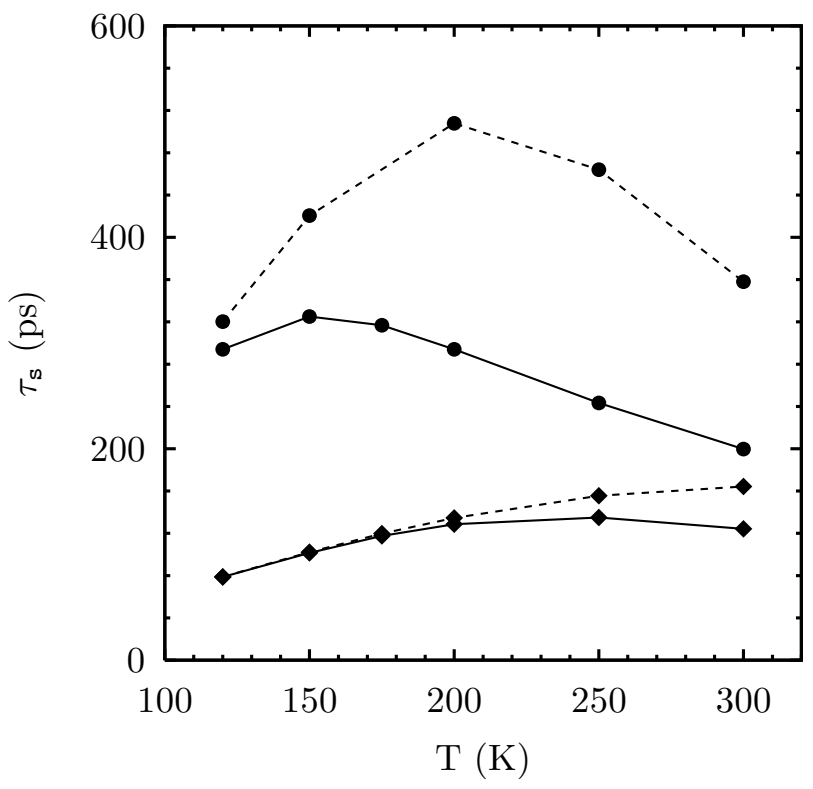

FIG. 3: SDT $\tau_{s}$ vs. the background temperature $T$ without the applied electric field for two QW's with width $a=17.8 \mathrm{~nm}$ $(\bullet)$ and $12.7 \mathrm{~nm}(\bullet)$. The solid curves are the SDT calculated with the lowest two subbands included and the dashed curves are those calculated with only the lowest subband.

the single subband approximation as dashed curves in order to reveal the contribution from the multi-subband effect. The figure shows that for wide QW, the SDT first increases with the background temperature and then decreases with it when the temperature rises to $150 \mathrm{~K}$. However, for narrow QW the SDT keeps increase until the temperature rises to about $250 \mathrm{~K}$ and then decreases slightly. The figure also shows the contribution of the higher subbands: For wide QW, the population of electrons in the higher subbands is larger even in the low temperature regime. As the temperature rises, the difference of the SDT of multi-subband model and single-subband one becomes larger and larger as the contribution of the higher subbands becomes more important. While for the narrow QW, the contribution of higher subbands is marginal when the temperature is lower than $250 \mathrm{~K}$, thus the SDT's from the multi- and single-subband models are almost the same in the regime $T<200 \mathrm{~K}$. After that, the SDT of multi-subband model becomes smaller than that of single-subband one. When the temperature rises higher than $250 \mathrm{~K}$, the SDT of multi-subband model decreases with $T$ while that of single-subband model keeps growing upto the room temperature.

The different behavior of the temperature dependence of the SDT at different temperature regime and for different well width originates from the DPT. In $n$-type GaAs QW's the dominant spin dephasing mechanism is the spin dephasing caused by the inhomogeneous broadening induced by the DPT, together with the spin conserving scattering. ${ }^{19,20}$ The increase of the temperature brings many effects on the spin dephasing through the DPT and the scattering rate: Firstly, the increase of temperature enhances the electron-phonon and the electron-electron scattering, which tends to drive electrons to a more homogeneous state, and thus reduces the spin dephasing. ${ }^{10}$ Nevertheless, the increase of the temperature also enhances the effect from the DPT. One can see from Eq. (2) that the DPT in the QW are composed of the terms linear and cubic in wave vector $\mathbf{k}=\left(k_{x}, k_{y}, 0\right)$. The effects from the both terms increase with temperature as electrons are driven to larger wave-vector states. This tends to bring a faster SDT.

For narrow QW's, the linear term is the dominate one even for the lowest subband as $\left\langle k_{z}^{2}\right\rangle$ is much larger than $k^{2}$ in the electron density and temperature regime of our investigation. Although the effect of the linear term increases with temperature, the increase rate is slower than that of of the scattering. Therefore in the single-subband model the SDT of the narrow QW keeps increasing with temperature. However, for wide QW's, the linear term of the lowest subband and the cubic term of the DPT are comparable. When the temperature rises to $200 \mathrm{~K}$, the contribution of cubic term becomes larger than that of the linear one of the lowest subband for the QW with $a=17.8 \mathrm{~nm}$. As the effect of the cubic term increases much faster with temperature than the scattering, the SDT decreases with temperature in the regime where the effect of the cubic term takes over that of the linear one.

When the contribution of the higher subbands are included, the increase of the temperature further enhances the DPT through exciting electrons to the higher subbands which brings the much larger DPT. As a result, for the QW with $a=17.8 \mathrm{~nm}$, the SDT increases only in the regime $T<150 \mathrm{~K}$ and then decreases when the effect of the multi-subband is included. Similarly, the SDT of the $12.7 \mathrm{~nm}$ QW no longer increases monotonically with the temperature but first increases with temperature and then decreases near room temperature.

We now study the effect of the applied electric field on the spin precession and spin dephasing. In Fig. 4, we plot the SDT as a function of the applied electric field for two temperatures and two well widths. It is seen from the figure that for low temperature, the SDT's first increase with $E$ for both wells just as the case in the single-subband model. ${ }^{8}$ With further increase of the electric field, $\tau_{s}$ of the wide $\mathrm{QW}$ decreases rapidly with $E$ once it is larger than $0.5 \mathrm{kV} / \mathrm{cm}$, while that of the narrow $\mathrm{QW}$ remains growing until $E$ is higher than 1 $\mathrm{kV} / \mathrm{cm}$. For high temperature, the SDT decreases with $E$ even in small electric field regime for the wide QW. While it is insensitive to the applied electric field in the regime $E<1 \mathrm{kV} / \mathrm{cm}$ for the narrow QW.

It is understood that the electric field can affect the spin dephasing in two competing ways: ${ }^{8}$ On one hand, when the electric field increases, the scattering rate increases due to the hot-electron effect. Consequently the electrons are driven to a more homogeneous state in $k$ space and thus the spin dephasing is reduced-Effect I; 


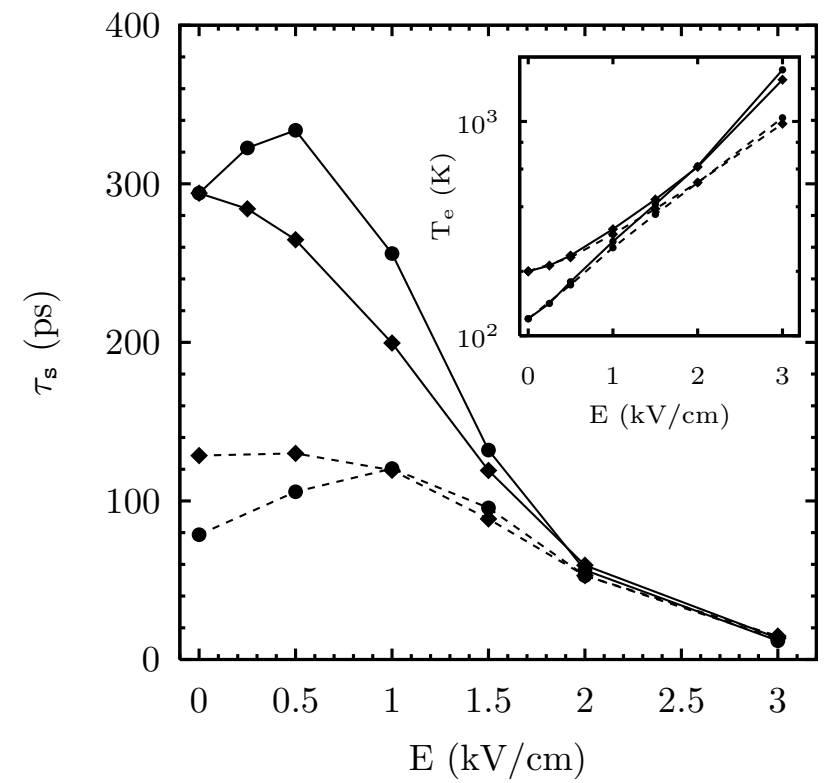

FIG. 4: SDT vs. the applied electric field $E$ at different temperatures and well widths: $\bullet, T=120 \mathrm{~K} ; \diamond, T=200 \mathrm{~K}$; Solid curves, $a=17.8 \mathrm{~nm}$; Dashed curves, $a=12.7 \mathrm{~nm}$. Inset: The corresponding electron temperature $T_{e}$ as a function of the electric field.

On the other hand, the applied electric field also drives the electrons to higher momentum states or higher subbands by rising the electron temperature and the drifting velocity and thus the spin dephasing is enhanced through the strengthening of the DPT-Effect II. In the low temperature and low applied electric field regime, where the linear DPT is more important and the electron drift velocity is small, the enhancement of the DP effect is relatively smaller and the reduction of the inhomogeneous broadening due to the hot-electron effect is more pronounced. Therefore the SDT is raised in this regime. However, when the electric field becomes larger, more electrons are driven to high momentum states where the cubic DPT dominates, moreover electrons are easier to be excited to the higher subbands where the linear DPT is much larger than that of the lowest subband, the second effect becomes more important and the SDT decreases with $E$ consequently.

For wide QW's, electrons are much easier to be excited to higher subbands and the domination of the cubic DPT is much easier to achieve than narrow QW's. Therefore at low temperature the SDT of the wide QW starts to decrease at relatively smaller electric field $(0.5 \mathrm{kV} / \mathrm{cm}$ in the figure). While the SDT of narrow QW keeps increasing until the electric field is $1 \mathrm{kV} / \mathrm{cm}$, which is about two times as large as that of the wide well. At high temperature, as the contribution from the cubic DPT is more important and also more electrons are excited to high subbands, Effect II is more important even at low electric field for wide QW's. As a result the SDT of wide QW decreases monotonically with the electric field. For the narrow QW in our calculation, both effects of the applied electric field is comparable in the regime $E<1 \mathrm{kV} / \mathrm{cm}$, consequently the SDT is insensitive to the electric field in this regime.

It is also interesting to note that at very high electric field regime $(E \geq 2 \mathrm{kV} / \mathrm{cm})$, the SDT is only determined by the electric field and is insensitive to the well width and the background temperature. In this regime, electrons are excited to high energy states by the electric field and the electron temperature $T_{e}$ is much higher than the background temperature $T$ as shown in the inset of Fig. 4. In this situation, the major scattering mechanism is the electron-electron Coulomb scattering and the dominant term of the DP term is the cubic one. Both rely on the electron temperature and are insensitive to the well width and the background temperature in the high electric field regime. Nevertheless it is noted that notwithstanding the fact that the electric field goes to very high in Fig. 4, the inter-valley scattering between $\Gamma$ - $L$ valleys, which plays important role in the high-field transport, is not included. Once this additional scattering is incorporated, the electron temperature will be reduced and the spin dephasing time may differ from what is predicted in Fig. 4 when $E \geq 2 \mathrm{kV} / \mathrm{cm}$. However a detailed study of this inter-valley effect is beyond the scope of this paper and also the spin dephasing mechanism in the $L$ (and also $X$ ) valley is not full understood to date.

In conclusion, we have studied the multi-subband effect on spin precession and spin dephasing in $n$-type GaAs QW's where electrons may occupy more than one subband. Our results show that due to the strong intersubband scattering, electrons hop frequently among the subbands and thus experience an average DP effective magnetic field regardless which subband the electrons are located. Therefore the spin precession and the spin dephasing of electrons in each subband are almost identical even though the DPT's in different subbands vary greatly. In the system we study, the spin dephasing is determined by the joint effects of the inhomogeneous broadening induced by the DPT and the spin-conserving scattering such as the electron-phonon and the electronelectron Coulomb scattering. The scattering tends to randomize electrons in the momentum space and reduces the inhomogeneous broadening and consequently leads to the rise of the SDT. Whereas the enhancement of the contribution of the DPT boosts the inhomogeneous broadening and therefore reduces the SDT. When the distribution of electrons varies due to the change of the well width, the background temperature and the applied electric field, the contributions from the linear and the cubic DPT's and the contribution from the higher subbands as well as the contribution from the spin conserving scattering change accordingly and the SDT varies consequently.

For narrow QW where electrons occupy only the lowest subband when the temperature is not too high, the dominant contribution from the DPT is the linear term. Therefore the SDT increases with temperature almost upto the room temperature as a result of the fast increase 
in the scattering rate and the relatively slower increase of effect from the linear DPT. While for wide QW, the contributions from the cubic DPT and the higher subbands are more important, the SDT only slightly increases with temperature in low temperature regime and then decreases with it. Similar effect also appears in the applied electric field dependence of the SDT. In low temperature and low electric field regime, the SDT increases with the electric field as the hot-electron effect effectively reduces the inhomogeneous broadening of the DPT. When the background temperature and/or the applied electric field increase, the SDT decreases as the contributions from the higher momentum states and high subbands become important. Whereas for wide QW's, as the contributions from the cubic DPT and the high subbands are more important, the SDT begins to decrease at smaller electric field than that of narrow QW's.

This work was supported by the Natural Science Foundation of China under Grant No. 90303012. MWW was also supported by the "100 Person Project" of Chinese Academy of Sciences and the Natural Science Foundation of China under Grant No. 10247002. MQW was partially supported by China Postdoctoral Science Foundation.
* Author to whom correspondence should be addressed; Electronic address: mwwu@ustc.edu.cn

$\dagger$ Mailing Address

1 S. A. Wolf, J. Supercond.: Incorping Novel Magnetism 13, 195 (2000).

2 M. Ziese and M. J. Thornton, eds., Spin Electronics (Springer, Berlin, 2001).

3 Y. A. Bychkov and E. I. Rashba, JETP Lett. 39, 78 (1984).

${ }^{4}$ Y. A. Bychkov and E. I. Rashba, J. Phys. C 17, 6039 (1984).

5 E. I. Rashba and A. L. Efros, Appl. Phys. Lett. 83, 5295 (2003).

6 S. Pramanik, S. Bandyopadhyay, and M. Cahay, Phys. Rev. B 68, 075313 (2003).

7 S. Pramanik, S. Bandyopadhyay, and M. Cahay, Appl. Phys. Lett. 84, 266 (2004).

8 M. Q. Weng, M. W. Wu, and L. Jiang, Phys. Rev. B 69 (2004), in Press.

9 A. Malinowski, R. S. Britton, T. Grevatt, R. T. Harley, D. A. Ritchie, and M. Y. Simmons, Phys. Rev. B 62, 13034 (2000).
10 M. Q. Weng and M. W. Wu, Phys. Rev. B 68, 075312 (2003).

11 M. I. D'yakonov and V. I. Perel', Zh. Eksp. Teor. Fiz. 60, 1954 (1971), [Sov. Phys.-JETP 33, 1053 (1971)].

12 A. P. Dmitriev, V. Y. Kachorovskii, M. S. Shur, and M. Stroscio, Solid State Commun. 113, 565 (2000).

13 G. Dresselhaus, Phys. Rev. 100, 580 (1955).

14 A. G. Aronov, G. E. Pikus, and A. N. Titkov, Zh. Eksp. Teor. Fiz. 84, 1170 (1983), [Sov. Phys.-JETP 57, 680 (1983)].

15 G. D. Mahan, Many-particle Physics (Plenum, New York, 1981).

${ }^{16}$ H. Haug and A. P. Jauho, Quantum Kinetics in Transport and Optics of Semiconductors (Springer-Verlag, Berlin, 1996).

17 M. W. Wu and H. Metiu, Phys. Rev. B 61, 2945 (2000).

18 T. Kuhn and F. Rossi, Phys. Rev. Lett. 69, 977 (1992).

19 M. W. Wu, J. Supercond.: Incorping Novel Magnetism 14, 245 (2001), cond-mat/0109258.

20 M. W. Wu and C. Z. Ning, Eur. Phys. J. B. 18, 373 (2000). 